\title{
Reliability Assessment of the Iraqi National Communication Network
}

\author{
Musaria K. Mahmood ${ }^{1}$, Fawzi M. Al-Naima², Zahraa Zaidan ${ }^{3}$ \\ ${ }^{1}$ Department of Electrical and Electronics Engineering, Istanbul Gelisim University, Turkey \\ ${ }^{2}$ Department of Computer Engineering, Al-Mamoon University College, Iraq \\ ${ }^{3}$ Department of Electrical Engineering, Tikrit University, Iraq
}

\begin{tabular}{l} 
Article Info \\
\hline Article history: \\
Received Aug 8, 2018 \\
Revised Sep 6, 2018 \\
Accepted Nov 19, 2018 \\
\hline
\end{tabular}

\section{Keyword:}

Backbone design

Clustering topology

Network reliability

WAN

\begin{abstract}
The design of communication networks continues to progress rapidly in more suitable forms to meet the challenges of the present era. This paper presents a tentative study of the design of a reliable Iraqi National Communication Network (INCN). The INCN connects all major cities in Iraq with Baghdad as the central node. The followed subjected procedure is a general method which can be applied to all similar problems concerning any Wide Area Network design. An optimal backbone is first designed by Prim's algorithm which has distances between cities as input data. The INCN is subjected to reliability improvement by adding links to the initial backbone. An improved algorithm based on tie-sets method is developed for network reliability computation. Three selected scenarios (Net1, Net2, and Net3) for the INCN are presented and underwent reliability estimation. Evaluation results show a successive improvement of the network reliability to yield to an optimal solution recognized as being Net3. The adopted topology for the INCN is based on two clusters having Baghdad as the common head cluster. Clustering will simplify the reliability evaluation by decreasing the number of tie-sets, and hence the computation complexity.
\end{abstract}

Copyright $@ 2018$ Institute of Advanced Engineering and Science. All rights reserved.

\section{Corresponding Author:}

Fawzi M. Al-Naima,

Department of Computer Engineering,

Al-Mamoon University College,

14 Ramadan St, Baghdad, Iraq.

Email: fawzi.alnaima@ieee.org

\section{INTRODUCTION}

Modern technology enables constructing societies to reach their public efficiently and with the high spreading area. Prosperity in such competitive universe requires a high quality and cost-effective exchange of data. This information exchange is only possible through optimum and reliable communication networks. Extensive works have been carried out in evaluating the reliability of wired and wireless networks [1]. Reliable communication networks play an essential role in managing and performing all the modern applications using heterogeneous networks [2]. The communication network demands better Quality of Services $(\mathrm{QoS})$ to perform the required tasks. Two of the most commonly used QoS metrics are network data flow and network reliability [3]. The reliability is defined as the probability that a network supports a given operation under certain conditions [4]. The probability of a system failure is considered as a random variable in reliability analysis. Due to the system complexity, edges failure is sometimes considered as being an independent event even when it is dependent [5].

There are three types of network reliability problems. The basic one is the two-terminal reliability as stated in [6]. The all-terminal problem is somewhat more difficult than the two-terminal reliability problem as presented in [7], while the K-terminal reliability is more comprehensive term depending on the value of (K) which can take any number from 2 to all nodes as depicted in [8]. The design of reliable communication 
networks is a critical subject for many applications. A typical reliable high performance, scalable, available and secure communications network for the next-generation electricity network is given in [9]. The design and simulation of a reliable data communication network for industrial plants is presented in [10]. The suggested work in [11] carries out a study of the mechanism design model of the communication network.

The INCN is planned to support various applications including data transmission, video, and voice application. The design of INCN is composed of two complementary parts. The first is the design of the optimal communication backbone by Prim's algorithm [12]. However, other methods for backbone design also exist as Kruskal Algorithm [13]. The second part represents a step by step improvement of the backbone towards a reliable topology of the network. The INCN is partitioned into two clusters resulting in a simplification in reliability evaluation. Clustering can be formulated as a multi-objective optimization problem for networks design problems [14]. Clusters are formed as groups of nodes taking into consideration the link structure in such a way that there should be many links within clusters and a minimum number of links between different clusters [15].

The tie-sets algorithm is one of the well-known methods for network reliability evaluation [16]. A new approach for deducing all minimal paths is introduced in the present research. This paper is organized as follows. Problem definition and network modeling are presented in the second section. In the third section a detailed description of the development of MTSA is given. The design methodology of different scenarios of the INCN, and the obtained results and discussion are given in section four. Also, the backbone tracing is outlined in this section. Finally, a brief conclusion is given in section five.

\section{PROBLEM FORMULATION}

Communication networks are usually modeled by a connected graph $\mathrm{G}=(N, L)$, with $(N)$ nodes, and $(L)$ Links [4]. $p_{i i}$ Presents the probability that a node $\left(n_{i}\right)$ is properly functioning, considered her as perfect $\left(p_{i i}=1\right)$, by the use of redundant materials. $p_{i k}$ is the probability of the link between node $n_{i}$ and node $n_{k}$. The link probability is considered as having a given value and is a direct function of link types and fabrication methods.

Communication network topology results from many factors such as the geographical location of nodes, communication activity, and obstacles (political, security, and natural). For the case study of INCN, the centers of eighteen provinces and two main cities (Samara, and Fallujah) are considered as main communication nodes as listed in Table 1.

Table 1. Major cities in Iraq

\begin{tabular}{ccccccccc}
\hline Node & Province & City & Node & Province & City & Node & Province & City \\
\hline 1 & Baghdad & Baghdad & 8 & Salah Aldin & Samara & 15 & Al Qadissia & Diwania \\
2 & Dohuk & Dohuk & 9 & Anbar & Ramadi & 16 & Muthana & Samawah \\
3 & Erbil & Erbil & 10 & Anbar & Falluja & 17 & Wasit & Kut \\
4 & Sulaymania & Sulaymania & 11 & Diyala & Baquba & 18 & Mayssan & Amarah \\
5 & Kirkuk & Kirkuk & 12 & Karbala & Karbala & 19 & Thikar & Nasiryah \\
6 & Ninava & Mosul & 13 & Babil & Hila & 20 & Basra & Basra \\
7 & Salah Aldin & Tikrit & 14 & Najaf & Najaf & & & \\
\hline
\end{tabular}

The principal node, i.e. node-1, is assigned to Baghdad city. It is used as the simplification node to reduce the topology to two basic sub-networks; the northern network $\left(\mathrm{C}_{1}\right)$, and the southern network $\left(\mathrm{C}_{2}\right)$. The graphical representation of a communication network is accomplished via matrix notation. The distance matrix $\mathrm{A}$ is an $(\mathrm{N} \times \mathrm{N})$ matrix, where each element $\left(a_{i k}\right)$ represents the distance in $\mathrm{km}$ between nodes $n_{i}$ and $n_{k}$. All diagonal elements are equal to zero $\left(a_{i i}=0\right)$. Distances for neighboring nodes are important for the backbone tracing algorithm, so other distances are marked by the word long distance (L) and set to a big numerical value for algorithm application. To simplify the presented information, the matrix A is presented as a table with (20) rows and (20) columns as shown in Table 2. 
Table 2. Distances in km between principal cities (matrix A)

\begin{tabular}{|c|c|c|c|c|c|c|c|c|c|c|c|c|c|c|c|c|c|c|c|c|}
\hline City & 1 & 2 & 3 & 4 & 5 & 6 & 7 & 8 & 9 & 10 & 11 & 12 & 13 & 14 & 15 & 16 & 17 & 18 & 19 & 20 \\
\hline 1 & 0 & $\bar{L}$ & $\mathrm{~L}$ & $\mathrm{~L}$ & $\mathrm{~L}$ & $\mathrm{~L}$ & 160 & 120 & 126 & 70 & 67 & 112 & 132 & 190 & 186 & $\bar{L}$ & 182 & $\bar{L}$ & $\mathrm{~L}$ & $\mathrm{~L}$ \\
\hline 2 & L & 0 & 158 & L & L & 73 & L & L & L & L & L & $\mathrm{L}$ & L & L & L & L & L & L & L & L \\
\hline 3 & $\bar{L}$ & 158 & 0 & 170 & 98 & 86 & $\bar{L}$ & $\bar{L}$ & $\bar{L}$ & $\vec{L}$ & $\bar{L}$ & $\mathrm{~L}$ & $\bar{L}$ & $\bar{L}$ & $\mathrm{~L}$ & $\mathrm{~L}$ & $\mathrm{~L}$ & $\mathrm{~L}$ & $\bar{L}$ & $\overrightarrow{\mathrm{L}}$ \\
\hline 4 & L & L & 180 & 0 & 111 & L & L & L & L & L & 278 & $\mathrm{~L}$ & L & L & L & L & $\mathrm{L}$ & $\mathrm{L}$ & L & $\mathrm{L}$ \\
\hline 5 & $\bar{L}$ & $\bar{L}$ & 98 & 111 & 0 & 177 & 129 & $\bar{L}$ & $\bar{L}$ & $\bar{L}$ & 229 & $\overline{\mathrm{L}}$ & $\bar{L}$ & $\bar{L}$ & $\bar{L}$ & $\bar{L}$ & $\mathrm{~L}$ & $\overline{\mathrm{L}}$ & $\bar{L}$ & $\bar{L}$ \\
\hline 6 & L & 73 & 86 & L & 177 & 0 & 220 & L & L & L & L & $\mathrm{L}$ & L & L & L & L & L & L & L & L \\
\hline 7 & 160 & $\mathrm{~L}$ & $\mathrm{~L}$ & $\bar{L}$ & 129 & 220 & 0 & 70 & 185 & $\overrightarrow{\mathrm{L}}$ & 163 & $\overrightarrow{\mathrm{L}}$ & L & $\bar{L}$ & $\bar{L}$ & L & L & $\bar{L}$ & $\mathrm{~L}$ & $\mathrm{~L}$ \\
\hline 8 & 120 & $\bar{L}$ & $\bar{L}$ & $\bar{L}$ & L & $\mathrm{L}$ & 70 & 0 & 110 & $\bar{L}$ & 100 & $\bar{L}$ & $\mathrm{~L}$ & $\mathrm{~L}$ & $\mathrm{~L}$ & $\mathrm{~L}$ & $\mathrm{~L}$ & $\mathrm{~L}$ & L & $\mathrm{L}$ \\
\hline 9 & 126 & $\mathrm{~L}$ & L & L & L & L & 185 & 110 & 0 & 50 & L & 141 & L & L & L & L & L & L & L & L \\
\hline 10 & 70 & L & L & L & L & L & L & L & 50 & 0 & L & L & L & L & L & L & L & L & L & L \\
\hline 11 & 67 & $\overline{\mathrm{L}}$ & $\bar{L}$ & 278 & 229 & $\overline{\mathrm{L}}$ & 163 & 100 & $\mathrm{~L}$ & L & 0 & $\overline{\mathrm{L}}$ & $\bar{L}$ & $\bar{L}$ & $\bar{L}$ & $\bar{L}$ & $\bar{L}$ & $\overline{\mathrm{L}}$ & $\bar{L}$ & $\overline{\mathrm{L}}$ \\
\hline 12 & 112 & $\mathrm{~L}$ & L & L & $\mathrm{L}$ & L & L & $\mathrm{L}$ & 141 & L & L & 0 & 48 & 79 & L & L & L & L & L & L \\
\hline 13 & 132 & L & $\mathrm{L}$ & L & L & L & L & L & L & L & L & 48 & 0 & 58 & 115 & L & 200 & L & L & L \\
\hline 14 & 190 & $\bar{L}$ & $\bar{L}$ & $\bar{L}$ & $\bar{L}$ & $\mathrm{~L}$ & $\bar{L}$ & $\mathrm{~L}$ & $\bar{L}$ & $\bar{L}$ & $\bar{L}$ & 79 & 58 & 0 & 83 & 145 & L & $\bar{L}$ & $\mathrm{~L}$ & $\bar{L}$ \\
\hline 15 & 186 & $\mathrm{~L}$ & $\mathrm{~L}$ & L & $\mathrm{L}$ & L & L & L & L & L & L & $\mathrm{L}$ & 115 & 83 & 0 & 107 & 160 & L & L & L \\
\hline 16 & $\mathrm{~L}$ & $\mathrm{~L}$ & L & L & L & L & L & L & L & L & L & $\mathrm{L}$ & $\mathrm{L}$ & 145 & 107 & 0 & L & L & 100 & L \\
\hline 17 & 182 & $\mathrm{~L}$ & $\mathrm{~L}$ & L & L & L & L & L & L & L & L & $\mathrm{L}$ & 200 & $\mathrm{~L}$ & 160 & L & 0 & 200 & 180 & L \\
\hline 18 & L & $\mathrm{L}$ & L & L & L & L & L & L & L & L & L & $\mathrm{L}$ & L & L & L & L & 200 & 0 & 140 & 175 \\
\hline 19 & $\bar{L}$ & $\overline{\mathrm{L}}$ & $\overline{\mathrm{L}}$ & $\bar{L}$ & $\bar{L}$ & $\bar{L}$ & $\bar{L}$ & $\bar{L}$ & $\bar{L}$ & $\bar{L}$ & $\bar{L}$ & $\overline{\mathrm{L}}$ & $\bar{L}$ & $\bar{L}$ & $\bar{L}$ & 100 & 180 & 140 & 0 & 240 \\
\hline 20 & L & L & $\mathrm{L}$ & L & L & L & L & L & L & L & L & L & L & L & L & L & L & 180 & 240 & 0 \\
\hline
\end{tabular}

\section{RESEARCH METHOD}

\subsection{Clustering simplification}

An algorithm based on tie-sets method is developed for the two-terminal reliability evaluation. The algorithm inputs are the commodity (source node- destination node), and links probability matrix. For mathematical argument, all links are assumed to be identical with probability $\mathrm{p}=0.9$. MTSA can be applied to a random topology without any loss of generality. The INCN case study has two clusters $(\mathrm{C} 1$ and $\mathrm{C} 2)$ connected via node-1. Two cases arise:

a. The commodity nodes are in the same cluster,

b. The commodity nodes are in different clusters.

This leads to a significant simplification, that is, instead of finding all tie-sets between any pair of nodes, it is only enough to find the tie-sets between a node and the central node. If commodity nodes are in the same cluster, the tie-sets between the source and the destination is calculated by considering only nodes inside this cluster, and the reliability can then directly be evaluated. If the source-destination pairs are in two different clusters, then, two tie-sets paths must be found. The first path is between the source node and the cluster head, i.e. node-1, whereas the second path is between the destination node and the cluster head. In such a case, the reliability from the source node to the destination node is simply calculated as the result of multiplying the two reliability values of the two paths.

\subsection{Implementation of the tie-sets process}

The tie-set generation process starts by denoting the source node $n_{s}$ and the destination node $n_{d}$, then seeking for all the possible paths connecting the pair $\left(n_{s}, n_{d}\right)$. A single route may be composed of one or a group of links and nodes. Matrix $T\left[t_{i k}\right]$ is formed by enumerating the $(N-1)$ ! combinations of the $\mathrm{N}$ node network (except $\left.n_{s}\right)$. The dimension of $T$ is $[(N-1) ! \times(N-1)]$, where the elements are node numbers. The matrix $P\left[p_{i k}\right]$ is the probability matrix where the element $p_{i k}$, is the probability of link between node $a_{i}$, and node $a_{k}$. The next step is to check whether each successive node-pair $\left(t_{r i}\right.$ and $\left.t_{r k}\right)$ in a row $(r)$ of the matrix $T$ is linked or not by consulting the corresponding value of $p_{i k}$ element in matrix $P$. An action is taken when there is no connection between nodes $n_{i}$ and $n_{k}$, in row $(r)$ of matrix $T$; that is $\left(p_{i k}=0\right)$ in the matrix $P$. We must then replace node $n_{k}$ by $n_{i}$. This measure ensures the correctness of the connection check operation since no possible connecting case will be skipped. After scanning all the successive elements of $T$, row-after-row the following simplification steps are taken:

a. Eliminate all the repeated nodes in one row,

b. Each node after the destination $\left(n_{d}\right)$ has to be removed, and

c. Keep one copy of similar tie-set.

Finally, we will get the collection of $(t)$ tie-sets $\left(T s_{1}, \ldots, T s_{t}\right)$, where the maximum number of links in a tie-set is equal to the number of nodes in the selected cluster as shown in Figure 1. The combinations number is related to the clusters and nodes numbers. The reliability can then be determined by the application of the Poincare inclusion equation given by [4]: 


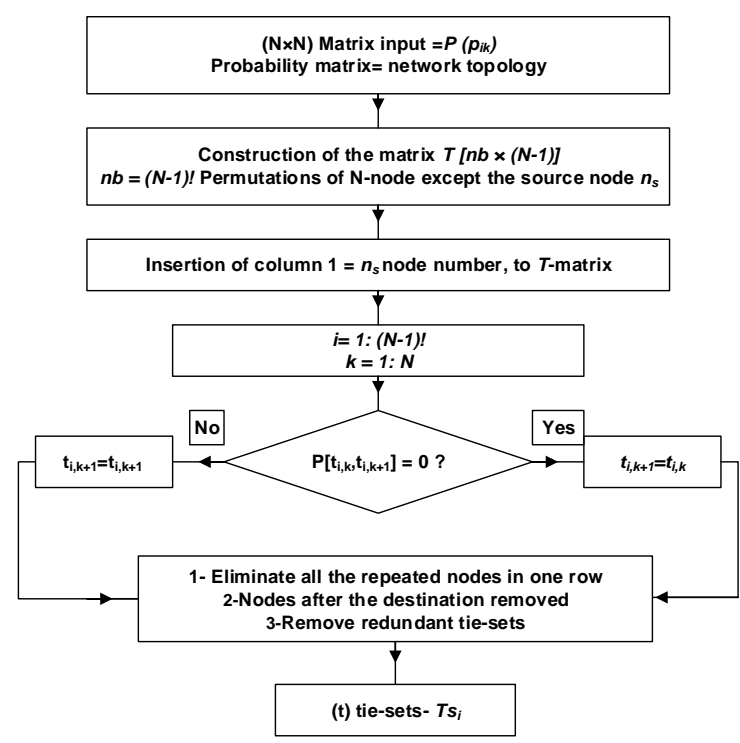

Figure 1. Generation of the $(t)$ tie-sets $\left(T s_{1}, \ldots, T s_{t}\right)$

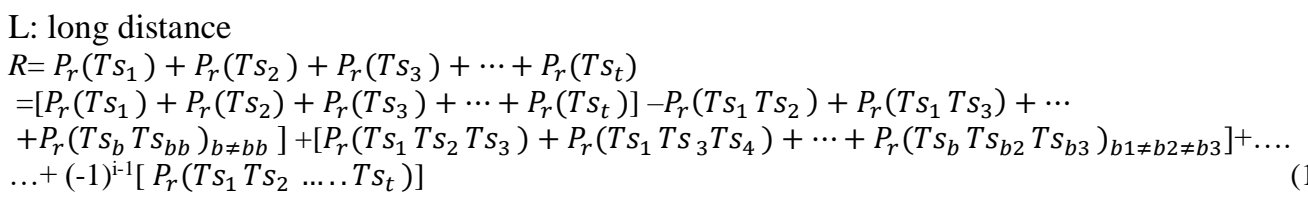

where $T s_{i}$ is the tie-set of order $i$ with $(1 \leq i \leq t) . P_{r}$, is the probability of tie-set equals to the multiplication of corresponding probabilities of all links in the selected tie-set. The flow chart of MTSA as depicted by Figure 2, begins by consulting a predefined vector (for each cluster) to identify the location of $n_{s}$ and $n_{d}$. If the two selected nodes (commodity) are in the same cluster, then the enumeration process of all tie-sets can begin. In such case the algorithm will only consider the nodes within the selected cluster. However, if the two nodes are in different clusters, then it is enough to enumerate the tie-sets between each node and the cluster head node-1. It follows that if $R_{1}$ is the reliability between $n_{s}$ and the cluster head and $R_{2}$ is the reliability between the cluster head and $n_{d}$, the total reliability $R_{s d}$ between $n_{s}$ and $n_{d}$ is then determined by:

$$
R_{s d}=R_{1} \times R_{2}
$$

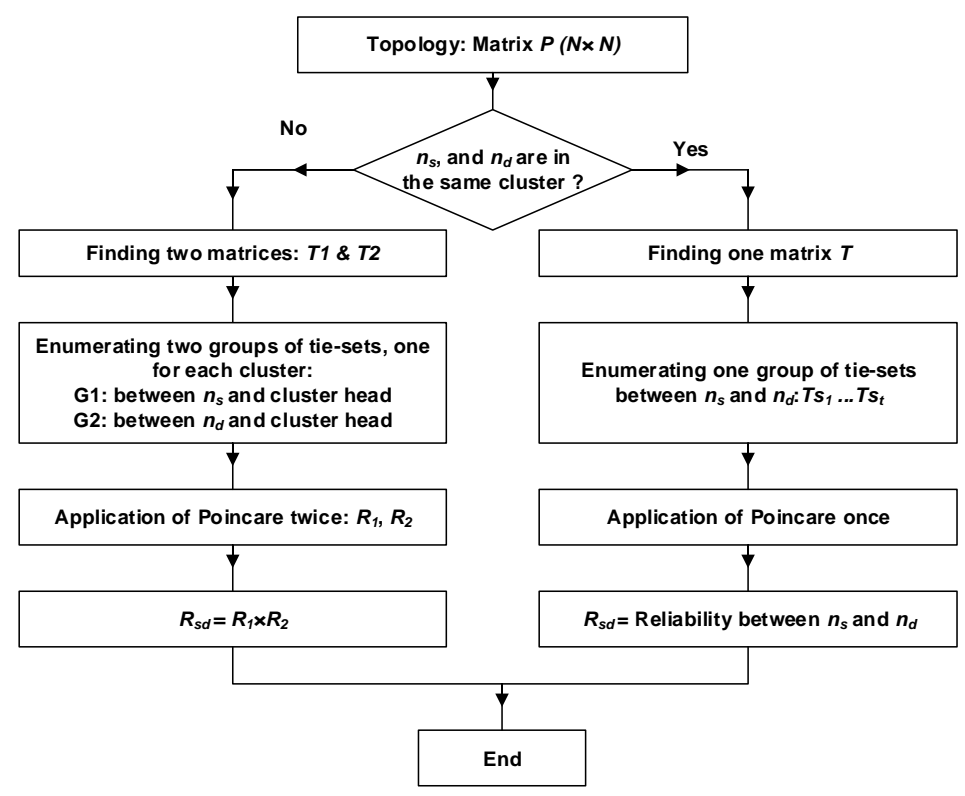

Figure 2. Flow chart of MTSA 


\section{RESULTS AND DISCUSSION}

\subsection{Backbone design}

For a network with N-nodes, the backbone is composed of (N-1) links. A Prim's algorithm is utilized as a tool for communication network backbone design. This algorithm is based on the enumeration of all distances as given in Table 3 in increasing order. The second step is to select sequentially links with the shortest distances provided that no closed loop is made in the topology. The process continues until (N-1) links have been selected. The unique exception is a link between Samara and Baghdad instead of Samara to Baquba. This is because the difference between the two distances is small $(<15 \mathrm{~km})$ and the route to Baquba requires a river traverse, rather there is a direct route between Samara and Baghdad. Table 3 summarizes the choice of (19) links required for network backbone while Figure 3 represents the network backbone.

Table 3. Backbone link selection

\begin{tabular}{cccccccccc}
\hline $\begin{array}{c}\text { Step } \\
\text { no }\end{array}$ & $\begin{array}{c}\text { Link } \\
\text { (edge) }\end{array}$ & $\begin{array}{c}\text { Distance } \\
\mathrm{km}\end{array}$ & $\begin{array}{c}\text { Selected } \\
\text { step }\end{array}$ & $\begin{array}{c}\text { Deleted } \\
\text { step }\end{array}$ & $\begin{array}{c}\text { Step } \\
\text { no }\end{array}$ & $\begin{array}{c}\text { Link } \\
\text { (edge) }\end{array}$ & $\begin{array}{c}\text { Distance } \\
\mathrm{km}\end{array}$ & $\begin{array}{c}\text { Selected } \\
\text { step }\end{array}$ & $\begin{array}{c}\text { Deleted } \\
\text { step }\end{array}$ \\
\hline 1 & $12-13$ & 48 & 1 & - & 17 & $1-12$ & 112 & 17 & - \\
2 & $9-10$ & 50 & 2 & - & 18 & $13-15$ & 115 & - & 18 \\
3 & $13-14$ & 58 & 3 & - & 19 & $1-8$ & 120 & 19 & - \\
4 & $7-8$ & 60 & 4 & - & 20 & $1-9$ & 126 & - & 20 \\
5 & $1-10$ & 65 & 5 & - & 21 & $5-7$ & 129 & 21 & - \\
6 & $1-11$ & 67 & 6 & - & 22 & $1-13$ & 132 & - & 22 \\
7 & $2-6$ & 73 & 7 & - & 23 & $18-19$ & 140 & 23 & - \\
8 & $12-14$ & 79 & - & 8 & 24 & $9-12$ & 141 & - & 24 \\
9 & $14-15$ & 83 & 9 & - & 25 & $14-16$ & 145 & - & 25 \\
10 & $3-6$ & 86 & 10 & - & 26 & $2-3$ & 158 & - & 26 \\
11 & $3-5$ & 98 & 11 & - & 27 & $15-17$ & 160 & 27 & - \\
12 & $16-19$ & 100 & 12 & - & 28 & $1-7$ & 160 & - & 28 \\
13 & $8-11$ & 100 & - & 13 & 29 & $7-11$ & 163 & - & 29 \\
14 & $8-9$ & 105 & - & 14 & 30 & $3-4$ & 170 & - & 30 \\
15 & $15-16$ & 107 & 15 & - & 31 & $18-20$ & 175 & 31 & - \\
16 & $4-5$ & 111 & 16 & - & & & & & \\
\hline
\end{tabular}

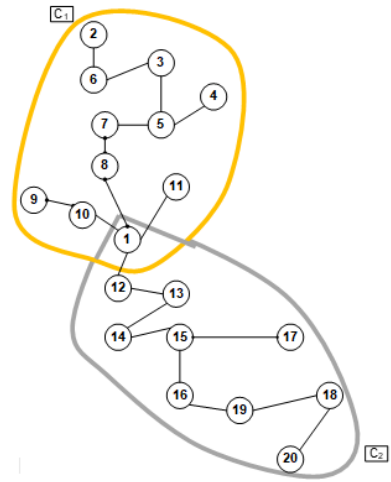

Figure 3. Communication network backbone

\subsection{Application of MTSA for proposed topologies}

The new approach for deducing all minimal paths enjoys the advantages of having a lower memory requirement since for a network of $\mathrm{N}$ nodes it depends on a combination number $c b \leq(N-1)$ ! rather than $N$ ! in the case of traditional tie-sets for all spanning trees. This decrease in $c b$, is positively reflected on the time needed for enumerating all tie-sets.

Clustering a network will significantly reduce the complexity of the application of (1) which is time consuming, and it is the principal source of computing delay. Breaking a network into several clusters will reduce the number of mathematical operations required to calculate (1). For example, if the nodes are divided into two equal groups, one for each cluster, the original N-node networks will be treated as two separated $\frac{N}{2}$ networks. This will facilitate the computing process by having a direct impact on the number of tie-sets within each cluster. Two $T$-matrices $\left(T_{1} \& T_{2}\right)$ will be found for the two clusters, each with dimension $\left[\left(\frac{N}{2}-1\right) ! \times\left(\frac{N}{2}-1\right)\right]$ instead of the original $T$-matrix of dimension $[(N-1) ! \times(N-1)]$. The required 
computation power for two $\left[\left(\frac{N}{2}-1\right) ! \times\left(\frac{N}{2}\right)\right]$ matrices is obviously less than that for one $[(N-1) ! \times(N-$ 1)] $T$-matrix. As an example, for 10-node network the $T$-matrix is of dimension $(362880 \times 9)$ while the two reduced matrices for each 5 -node cluster are of dimension $(24 \times 4)$. That is, the combination number is decreased by a factorial factor.

Clustering a network and the use of the newly developed mechanism for deducing tie-sets will contribute together to simplify the network reliability evaluation. The simplification ratio will depend on the number of clusters, the nodes number in each cluster, and the original and subnetwork topologies. Added to that, there is no need for Boolean algebra application, and that, MTSA handles both directed and undirected links. The proposed MTSA is proven to be faster than the traditional tie-sets algorithm which enables it to be used as a real-time reliability evaluation tool.

Based on the INCN backbone, three selected topologies are proposed as shown in Figure 4. The choices of these topologies are made after reviewing the geographical locations of nodes and adding some links to improve the reliability with minimum additional cost. Other additional topologies can be proposed for reliability evaluation keeping in mind the cost introduced by the addition of new links to the original backbone. It should be emphasized that the present research is a methodical case study that can be utilized as a platform for future analysis to make the correct choice of optimal topology.

The chosen topologies have been subjected to reliability evaluation using MTSA. The obtained results are given for all commodities (source- destination) in Table 4 in appendix, where $\mathrm{S}$ is the source node, $\mathrm{D}$ is the destination node, and $\mathrm{R}$ is the two-terminal reliability. These results show an increasing improvement in the network reliability in ascending order from Net1, Net2, to Net3. The average reliability is taken as a measuring criterion for evaluating different topologies. Net3 shows a remarkable improvement in the value of average reliability. The average values of all two-terminal reliabilities are found to be $(0.932947)$ for Net1, $(0.9416658)$ for Net2, and $(0.9619857)$ for Net3. This is can be explained by the addition of more links to the other candidate topologies.

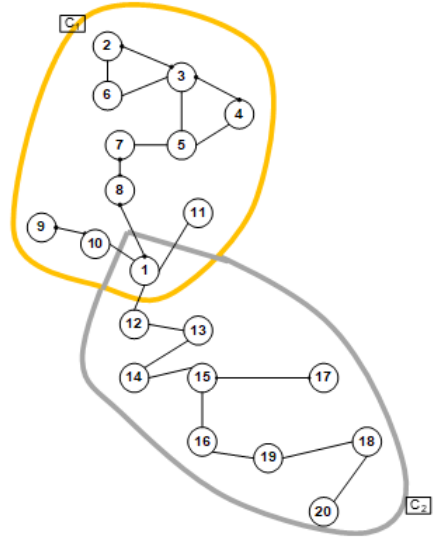

(a) Net1

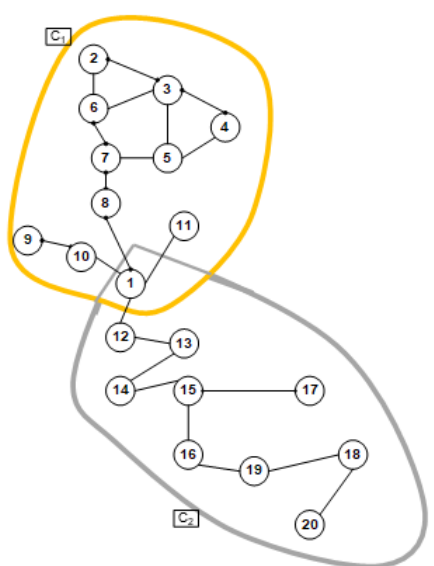

(b) Net2

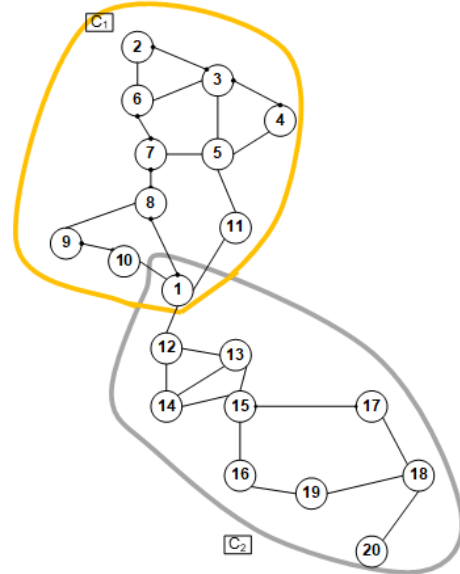

(c) Net3

Figure 4. Candidate topologies

\section{CONCLUSION}

Four phases are to be followed to ensure an optimal communication network, namely, specifications, design, simulation, and implementation. The presented work concentrates on the design procedure of a communication network which is based on the specifications and geographical locations of main communication nodes. Simulation scenarios must be employed to validate the expected results before the implementation of the real network. The design of network backbone using Prim's algorithm is part of the present research. Many selected scenarios have been subjected to reliability evaluation using a newly developed procedure (MTSA). One of the proposed candidate networks has been selected as the best reliable network for the case study of INCN. The MTSA is based on tie-sets algorithm, with a new proposed method to find tie sets group. Network clustering leads to a large reduction in the size of tie-sets matrix which contributes toward the simplification of MTSA application. The procedure formulated in this paper promises to be an adequate tool in the design of reliable networks, which may be used as an important background for any computer network or any other similar communication network. 


\section{REFERENCES}

[1] Panda DK, Dash RK., "Reliability Evaluation and Analysis of Mobile Ad Hoc Networks," International Journal of Electrical and Computer Engineering (IJECE), vol. 7(1), pp. 479-485, 2017.

[2] Sun H, Cai X, Chenc H., "Study on Parameter Evaluation System of Communication Network. Procedia Computer Science," vol. 107, pp. 584- 589, 2017.

[3] Yong LK, Soh S, Rai S., "Computer Communication Network Upgrade for Optimal Capacity Related Reliability," Asia-Pacific Conference on Communications, Perth, Western Australia, pp. 102-106, 2005.

[4] Mahmood, MK, Al-Naima, F, Abdulla, L., "An Efficient Multi-stages Algorithm for the Determination of Communication Network Reliability," International Journal of Computers and Communication, vol. 9, pp. 36-43, 2015.

[5] Yang X, Liu Y, Mi C, Tang C., "System Reliability Analysis through Active Learning Kriging Model with Truncated Candidate Region," Reliability Engineering and System Safety, vol. 169, pp. 235-241, 2018.

[6] Majid F, Luiz HB., "Finding all the Lower Boundary Points in a Multi-state Two-terminal Network," IEEE Trans. on Reliability, vol. 66, pp. 677-688, 2017.

[7] Willem P, Teresa G, Robert K., "A Comparison between Two All-terminal Reliability Algorithms," Journal of Advances in Computer Networks, vol. 3(4), pp. 284-290, 2015.

[8] Chatterjee S, Venkata RB, Gajendra KV, Verma A., "An Improved Algorithm for K-terminal Probabilistic Network Reliability Analysis," Journal of Reliability and Statistical Studies, vol. 10(1), pp. 15-26, 2017.

[9] Kenneth CB, Jayant GD, Tewfik LD, Mark M., "Communication Network Architecture and Design Principles for Smart Grids," Bell Labs Technical Journal, vol. 15(2), 2010.

[10] Mahmood MK, Al-Naima F, Uzunoglu N., "Design and Simulation of a Data Transmission Network for Industrial Control System Subject to Reliability Improvement," ICFCN, Bahgdad, 2012.

[11] Ludovic R, Tristan T., "Mechanism design and communication networks", Theoretical Economics, vol. 7(3), pp. 489-533, 2012.

[12] Dey A, Pal A., "Prim's Algorithm for Solving Minimum Spanning Tree Problem in Fuzzy Environment," Annals of Fuzzy Mathematics and Informatics, vol. 12(3), pp. 419-430, 2016.

[13] Li H, Xia Q, Wang Y., "Research and Improvement of Kruskal Algorithm. Journal of Computer and Communications," vol. 5, pp. 63-69, 2017.

[14] Virmani C, Pillai A, Juneja D., "Clustering in Aggregated User Profiles across Multiple Social Network," International Journal of Electrical and Computer Engineering (IJECE), vol. 7(6), pp. 3692-3699, 2017.

[15] Schaeffer S., "Graph Clustering," Computer Science Review, vol. 1, pp. 27-64.2007.

[16] Mahmood MK, "Development of a new algorithm for communication networks reliability based on tie-set method combined with a modified flooding algorithm," Tikrit Journal of Engineering Sciences, vol. 20, pp. 10-20, 2013. 


\section{APPENDIX}

Table 4. Reliability evaluation based on MTSA (a) Net1 results

\begin{tabular}{|c|c|c|c|c|c|c|c|c|c|c|c|}
\hline $\mathrm{S}$ & $\mathrm{D}$ & $\mathrm{R}$ & $\mathrm{S}$ & D & $\mathrm{R}$ & $\mathrm{S}$ & $\mathrm{D}$ & $\mathrm{R}$ & $\mathrm{S}$ & $\mathrm{D}$ & $\mathrm{R}$ \\
\hline 1 & 2 & 0.9604 & 3 & 16 & 0.9205 & 6 & 19 & 0.9097 & 11 & 13 & 0.9232 \\
\hline 1 & 3 & 0.9693 & 3 & 17 & 0.9327 & 6 & 20 & 0.8187 & 11 & 14 & 0.9169 \\
\hline 1 & 4 & 0.9593 & 3 & 18 & 0.9125 & 7 & 8 & 0.9968 & 11 & 15 & 0.9129 \\
\hline 1 & 5 & 0.9685 & 3 & 18 & 0.9125 & 7 & 9 & 0.9756 & 11 & 16 & 0.9260 \\
\hline 1 & 6 & 0.9706 & 3 & 19 & 0.9084 & 7 & 10 & 0.9730 & 11 & 17 & 0.9383 \\
\hline 1 & 7 & 0.9890 & 3 & 20 & 0.8176 & 7 & 11 & 0.9706 & 11 & 18 & 0.9179 \\
\hline 1 & 8 & 0.9917 & 4 & 5 & 0.9872 & 7 & 12 & 0.9517 & 11 & 19 & 0.9139 \\
\hline 1 & 9 & 0.9758 & 4 & 6 & 0.9817 & 7 & 13 & 0.9364 & 11 & 20 & 0.8225 \\
\hline 1 & 10 & 0.9785 & 4 & 7 & 0.9620 & 7 & 14 & 0.9300 & 12 & 13 & 0.9800 \\
\hline 1 & 11 & 0.9751 & 4 & 8 & 0.9610 & 7 & 15 & 0.9259 & 12 & 14 & 0.9747 \\
\hline 1 & 12 & 0.9622 & 4 & 9 & 0.9424 & 7 & 16 & 0.9392 & 12 & 15 & 0.9677 \\
\hline 1 & 13 & 0.9468 & 4 & 10 & 0.9418 & 7 & 17 & 0.9517 & 12 & 16 & 0.9789 \\
\hline 1 & 14 & 0.9404 & 4 & 11 & 0.9642 & 7 & 18 & 0.9310 & 12 & 17 & 0.9414 \\
\hline 1 & 15 & 0.9362 & 4 & 12 & 0.9231 & 7 & 19 & 0.9269 & 12 & 18 & 0.9372 \\
\hline 1 & 16 & 0.9497 & 4 & 13 & 0.9083 & 7 & 20 & 0.8342 & 12 & 19 & 0.9497 \\
\hline 1 & 17 & 0.9622 & 4 & 14 & 0.9021 & 8 & 9 & 0.9785 & 12 & 20 & 0.8547 \\
\hline 1 & 18 & 0.9414 & 4 & 15 & 0.8981 & 8 & 10 & 0.9758 & 13 & 14 & 0.9619 \\
\hline 1 & 19 & 0.9372 & 4 & 16 & 0.9110 & 8 & 11 & 0.9716 & 13 & 15 & 0.9619 \\
\hline 1 & 20 & 0.8435 & 4 & 17 & 0.9231 & 8 & 12 & 0.9543 & 13 & 16 & 0.9800 \\
\hline 2 & 3 & 0.9872 & 4 & 18 & 0.9031 & 8 & 13 & 0.9390 & 13 & 17 & 0.9303 \\
\hline 2 & 4 & 0.9747 & 4 & 19 & 0.8991 & 8 & 14 & 0.9326 & 13 & 18 & 0.9303 \\
\hline 2 & 5 & 0.9816 & 4 & 20 & 0.8092 & 8 & 15 & 0.9285 & 13 & 19 & 0.9468 \\
\hline 2 & 6 & 0.9872 & 5 & 6 & 0.9888 & 8 & 16 & 0.9418 & 13 & 20 & 0.8521 \\
\hline 2 & 7 & 0.9642 & 5 & 7 & 0.9706 & 8 & 17 & 0.9543 & 14 & 15 & 0.9747 \\
\hline 2 & 8 & 0.9629 & 5 & 8 & 0.9698 & 8 & 18 & 0.9336 & 14 & 16 & 0.9677 \\
\hline 2 & 9 & 0.9440 & 5 & 9 & 0.9511 & 8 & 19 & 0.9295 & 14 & 17 & 0.9226 \\
\hline 2 & 10 & 0.9432 & 5 & 10 & 0.9507 & 8 & 20 & 0.8365 & 14 & 18 & 0.9212 \\
\hline 2 & 11 & 0.9619 & 5 & 11 & 0.9759 & 9 & 10 & 0.9785 & 14 & 19 & 0.9362 \\
\hline 2 & 12 & 0.9241 & 5 & 12 & 0.9319 & 9 & 11 & 0.9545 & 14 & 20 & 0.8426 \\
\hline 2 & 13 & 0.9093 & 5 & 13 & 0.9170 & 9 & 12 & 0.9389 & 15 & 16 & 0.9747 \\
\hline 2 & 14 & 0.9031 & 5 & 14 & 0.9108 & 9 & 13 & 0.9239 & 15 & 17 & 0.9212 \\
\hline 2 & 15 & 0.8991 & 5 & 15 & 0.9067 & 9 & 14 & 0.9176 & 15 & 18 & 0.9226 \\
\hline 2 & 16 & 0.9120 & 5 & 16 & 0.9198 & 9 & 15 & 0.9135 & 15 & 19 & 0.9404 \\
\hline 2 & 17 & 0.9241 & 5 & 17 & 0.9319 & 9 & 16 & 0.9267 & 15 & 20 & 0.8463 \\
\hline 2 & 18 & 0.9048 & 5 & 18 & 0.9117 & 9 & 17 & 0.9389 & 16 & 17 & 0.9372 \\
\hline 2 & 19 & 0.9001 & 5 & 19 & 0.9077 & 9 & 18 & 0.9186 & 16 & 18 & 0.9414 \\
\hline 2 & 20 & 0.8101 & 5 & 20 & 0.8169 & 9 & 19 & 0.9145 & 16 & 19 & 0.9622 \\
\hline 3 & 4 & 0.9872 & 6 & 7 & 0.9751 & 9 & 20 & 0.8231 & 16 & 20 & 0.8660 \\
\hline 3 & 5 & 0.9941 & 6 & 8 & 0.9737 & 10 & 11 & 0.9556 & 17 & 18 & 0.9622 \\
\hline 3 & 6 & 0.9941 & 6 & 9 & 0.9544 & 10 & 12 & 0.9416 & 17 & 19 & 0.9414 \\
\hline 3 & 7 & 0.9725 & 6 & 10 & 0.9534 & 10 & 13 & 0.9265 & 17 & 20 & 0.8472 \\
\hline 3 & 8 & 0.9714 & 6 & 11 & 0.9706 & 10 & 14 & 0.9202 & 18 & 19 & 0.9622 \\
\hline 3 & 9 & 0.9525 & 6 & 12 & 0.9340 & 10 & 15 & 0.9161 & 18 & 20 & 0.8660 \\
\hline 3 & 10 & 0.9517 & 6 & 13 & 0.9190 & 10 & 16 & 0.9293 & 19 & 20 & 0.9000 \\
\hline 3 & 11 & 0.9725 & 6 & 14 & 0.9128 & 10 & 17 & 0.9416 & & & \\
\hline 3 & 12 & 0.9327 & 6 & 15 & 0.9087 & 10 & 18 & 0.9212 & & & \\
\hline 3 & 13 & 0.9177 & 6 & 16 & 0.9218 & 10 & 19 & 0.9171 & & & \\
\hline 3 & 14 & 0.9115 & 6 & 17 & 0.9340 & 10 & 20 & 0.8254 & & & \\
\hline 3 & 15 & 0.9074 & 6 & 18 & 0.9137 & 11 & 12 & 0.9383 & & & \\
\hline \multicolumn{12}{|c|}{ Average reliability $=0.9330$} \\
\hline
\end{tabular}

$\mathrm{S}=$ Sender node, $\mathrm{D}=$ Destination Node, $\mathrm{R}=$ Reliability 
(b) Net2 results

\begin{tabular}{|c|c|c|c|c|c|c|c|c|c|c|c|}
\hline $\mathrm{S}$ & $\mathrm{D}$ & $\mathrm{R}$ & $\mathrm{S}$ & $\mathrm{D}$ & $\mathrm{R}$ & $\mathrm{S}$ & $\mathrm{D}$ & $\mathrm{R}$ & $\mathrm{S}$ & $\mathrm{D}$ & $\mathrm{R}$ \\
\hline 1 & 2 & 0.9782 & 3 & 16 & 0.9389 & 6 & 19 & 0.9254 & 11 & 13 & 0.9331 \\
\hline 1 & 3 & 0.9886 & 3 & 17 & 0.9513 & 6 & 20 & 0.8329 & 11 & 14 & 0.9268 \\
\hline 1 & 4 & 0.9798 & 3 & 18 & 0.9307 & 7 & 8 & 0.9978 & 11 & 15 & 0.9227 \\
\hline 1 & 5 & 0.9906 & 3 & 19 & 0.9266 & 7 & 9 & 0.9773 & 11 & 16 & 0.9359 \\
\hline 1 & 6 & 0.9874 & 3 & 20 & 0.8339 & 7 & 10 & 0.9756 & 11 & 17 & 0.9483 \\
\hline 1 & 7 & 0.9926 & 4 & 5 & 0.9888 & 7 & 11 & 0.9851 & 11 & 18 & 0.9278 \\
\hline 1 & 8 & 0.9943 & 4 & 6 & 0.9865 & 7 & 12 & 0.9551 & 11 & 19 & 0.9236 \\
\hline 1 & 9 & 0.9774 & 4 & 7 & 0.9863 & 7 & 13 & 0.9398 & 11 & 20 & 0.8313 \\
\hline 1 & 10 & 0.9793 & 4 & 8 & 0.9843 & 7 & 14 & 0.9334 & 12 & 13 & 0.9800 \\
\hline 1 & 11 & 0.9855 & 4 & 9 & 0.9642 & 7 & 15 & 0.9293 & 12 & 14 & 0.9747 \\
\hline 1 & 12 & 0.9622 & 4 & 10 & 0.9627 & 7 & 16 & 0.9427 & 12 & 15 & 0.9677 \\
\hline 1 & 13 & 0.9468 & 4 & 11 & 0.9746 & 7 & 17 & 0.9551 & 12 & 16 & 0.9789 \\
\hline 1 & 14 & 0.9404 & 4 & 12 & 0.9428 & 7 & 18 & 0.9344 & 12 & 17 & 0.9414 \\
\hline 1 & 15 & 0.9362 & 4 & 13 & 0.9276 & 7 & 19 & 0.9303 & 12 & 18 & 0.9372 \\
\hline 1 & 16 & 0.9497 & 4 & 14 & 0.9213 & 7 & 20 & 0.8373 & 12 & 19 & 0.9497 \\
\hline 1 & 17 & 0.9622 & 4 & 15 & 0.9172 & 8 & 9 & 0.9793 & 12 & 20 & 0.8547 \\
\hline 1 & 18 & 0.9414 & 4 & 16 & 0.9305 & 8 & 10 & 0.9774 & 13 & 14 & 0.9619 \\
\hline 1 & 19 & 0.9372 & 4 & 17 & 0.9428 & 8 & 11 & 0.9850 & 13 & 15 & 0.9619 \\
\hline 1 & 20 & 0.8435 & 4 & 18 & 0.9223 & 8 & 12 & 0.9568 & 13 & 16 & 0.9800 \\
\hline 2 & 3 & 0.9888 & 4 & 19 & 0.9182 & 8 & 13 & 0.9415 & 13 & 17 & 0.9303 \\
\hline 2 & 4 & 0.9778 & 4 & 20 & 0.8264 & 8 & 14 & 0.9351 & 13 & 18 & 0.9303 \\
\hline 2 & 5 & 0.9865 & 5 & 6 & 0.9955 & 8 & 15 & 0.9309 & 13 & 19 & 0.9468 \\
\hline 2 & 6 & 0.9888 & 5 & 7 & 0.9971 & 8 & 16 & 0.9443 & 13 & 20 & 0.8521 \\
\hline 2 & 7 & 0.9848 & 5 & 8 & 0.9951 & 8 & 17 & 0.9568 & 14 & 15 & 0.9747 \\
\hline 2 & 8 & 0.9828 & 5 & 9 & 0.9748 & 8 & 18 & 0.9361 & 14 & 16 & 0.9677 \\
\hline 2 & 9 & 0.9628 & 5 & 10 & 0.9733 & 8 & 19 & 0.9319 & 14 & 17 & 0.9226 \\
\hline 2 & 10 & 0.9612 & 5 & 11 & 0.9855 & 8 & 20 & 0.8387 & 14 & 18 & 0.9212 \\
\hline 2 & 11 & 0.9727 & 5 & 12 & 0.9531 & 9 & 10 & 0.9793 & 14 & 19 & 0.9362 \\
\hline 2 & 12 & 0.9413 & 5 & 13 & 0.9379 & 9 & 11 & 0.9666 & 14 & 20 & 0.8426 \\
\hline 2 & 13 & 0.9262 & 5 & 14 & 0.9315 & 9 & 12 & 0.9405 & 15 & 16 & 0.9747 \\
\hline 2 & 14 & 0.9199 & 5 & 15 & 0.9274 & 9 & 13 & 0.9254 & 15 & 17 & 0.9212 \\
\hline 2 & 15 & 0.9158 & 5 & 16 & 0.9407 & 9 & 14 & 0.9191 & 15 & 18 & 0.9226 \\
\hline 2 & 16 & 0.9290 & 5 & 17 & 0.9531 & 9 & 15 & 0.9151 & 15 & 19 & 0.9404 \\
\hline 2 & 17 & 0.9413 & 5 & 18 & 0.9325 & 9 & 16 & 0.9282 & 15 & 20 & 0.8463 \\
\hline 2 & 18 & 0.9209 & 5 & 19 & 0.9284 & 9 & 17 & 0.9405 & 16 & 17 & 0.9372 \\
\hline 2 & 19 & 0.9168 & 5 & 20 & 0.8355 & 9 & 18 & 0.9201 & 16 & 18 & 0.9414 \\
\hline 2 & 20 & 0.8251 & 6 & 7 & 0.9941 & 9 & 19 & 0.9161 & 16 & 19 & 0.9622 \\
\hline 3 & 4 & 0.9888 & 6 & 8 & 0.9921 & 9 & 20 & 0.8245 & 16 & 20 & 0.8660 \\
\hline 3 & 5 & 0.9974 & 6 & 9 & 0.9719 & 10 & 11 & 0.9668 & 17 & 18 & 0.9622 \\
\hline 3 & 6 & 0.9974 & 6 & 10 & 0.9703 & 10 & 12 & 0.9423 & 17 & 19 & 0.9414 \\
\hline 3 & 7 & 0.9953 & 6 & 11 & 0.9817 & 10 & 13 & 0.9272 & 17 & 20 & 0.8472 \\
\hline 3 & 8 & 0.9932 & 6 & 12 & 0.9501 & 10 & 14 & 0.9209 & 18 & 19 & 0.9622 \\
\hline 3 & 9 & 0.9730 & 6 & 13 & 0.9349 & 10 & 15 & 0.9168 & 18 & 20 & 0.8660 \\
\hline 3 & 10 & 0.9715 & 6 & 14 & 0.9285 & 10 & 16 & 0.9300 & 19 & 20 & 0.9000 \\
\hline 3 & 11 & 0.9833 & 6 & 15 & 0.9244 & 10 & 17 & 0.9423 & & & \\
\hline 3 & 12 & 0.9513 & 6 & 16 & 0.9377 & 10 & 18 & 0.9219 & & & \\
\hline 3 & 13 & 0.9361 & 6 & 17 & 0.9501 & 10 & 19 & 0.9178 & & & \\
\hline 3 & 14 & 0.9297 & 6 & 18 & 0.9295 & 10 & 20 & 0.8260 & & & \\
\hline 3 & 15 & 0.9256 & & & & 11 & 12 & 0.9483 & & & \\
\hline \multicolumn{12}{|c|}{ Average reliability $=0.9417$} \\
\hline
\end{tabular}

$\mathrm{S}=$ Sender node, $\mathrm{D}=$ Destination Node, $\mathrm{R}=$ Reliability 
(c) Net3 results

\begin{tabular}{|c|c|c|c|c|c|c|c|c|c|c|c|}
\hline $\mathrm{S}$ & $\mathrm{D}$ & $\mathrm{R}$ & $\mathrm{S}$ & $\mathrm{D}$ & $\mathrm{R}$ & $S$ & $\mathrm{D}$ & $\mathrm{R}$ & $\mathrm{S}$ & $\mathrm{D}$ & $\mathrm{R}$ \\
\hline 1 & 2 & 0.9782 & 3 & 16 & 0.9526 & 6 & 19 & 0.9438 & 11 & 13 & 0.9506 \\
\hline 1 & 3 & 0.9886 & 3 & 17 & 0.9583 & 6 & 20 & 0.9347 & 11 & 14 & 0.9431 \\
\hline 1 & 4 & 0.9798 & 3 & 18 & 0.9454 & 7 & 8 & 0.9978 & 11 & 15 & 0.9496 \\
\hline 1 & 5 & 0.9906 & 3 & 19 & 0.9450 & 7 & 9 & 0.9773 & 11 & 16 & 0.9496 \\
\hline 1 & 6 & 0.9874 & 3 & 20 & 0.9359 & 7 & 10 & 0.9756 & 11 & 17 & 0.9553 \\
\hline 1 & 7 & 0.9926 & 4 & 5 & 0.9888 & 7 & 11 & 0.9851 & 11 & 18 & 0.9424 \\
\hline 1 & 8 & 0.9943 & 4 & 6 & 0.9865 & 7 & 12 & 0.9621 & 11 & 19 & 0.9420 \\
\hline 1 & 9 & 0.9774 & 4 & 7 & 0.9863 & 7 & 13 & 0.9575 & 11 & 20 & 0.9329 \\
\hline 1 & 10 & 0.9793 & 4 & 8 & 0.9843 & 7 & 14 & 0.9499 & 12 & 13 & 0.9909 \\
\hline 1 & 11 & 0.9855 & 4 & 9 & 0.9642 & 7 & 15 & 0.9565 & 12 & 14 & 0.9851 \\
\hline 1 & 12 & 0.9693 & 4 & 10 & 0.9627 & 7 & 16 & 0.9564 & 12 & 15 & 0.9897 \\
\hline 1 & 13 & 0.9646 & 4 & 11 & 0.9746 & 7 & 17 & 0.9621 & 12 & 16 & 0.9873 \\
\hline 1 & 14 & 0.9570 & 4 & 12 & 0.9497 & 7 & 18 & 0.9492 & 12 & 17 & 0.9442 \\
\hline 1 & 15 & 0.9636 & 4 & 13 & 0.9451 & 7 & 19 & 0.9487 & 12 & 18 & 0.9417 \\
\hline 1 & 16 & 0.9636 & 4 & 14 & 0.9376 & 7 & 20 & 0.9396 & 12 & 19 & 0.9560 \\
\hline 1 & 17 & 0.9693 & 4 & 15 & 0.9441 & 8 & 9 & 0.9793 & 12 & 20 & 0.9527 \\
\hline 1 & 18 & 0.9563 & 4 & 16 & 0.9440 & 8 & 10 & 0.9774 & 13 & 14 & 0.9838 \\
\hline 1 & 19 & 0.9558 & 4 & 17 & 0.9497 & 8 & 11 & 0.9850 & 13 & 15 & 0.9962 \\
\hline 1 & 20 & 0.9466 & 4 & 18 & 0.9369 & 8 & 12 & 0.9638 & 13 & 16 & 0.9942 \\
\hline 2 & 3 & 0.9888 & 4 & 19 & 0.9365 & 8 & 13 & 0.9592 & 13 & 17 & 0.9416 \\
\hline 2 & 4 & 0.9778 & 4 & 20 & 0.9275 & 8 & 14 & 0.9515 & 13 & 18 & 0.9422 \\
\hline 2 & 5 & 0.9865 & 5 & 6 & 0.9955 & 8 & 15 & 0.9581 & 13 & 19 & 0.9597 \\
\hline 2 & 6 & 0.9888 & 5 & 7 & 0.9971 & 8 & 16 & 0.9581 & 13 & 20 & 0.9549 \\
\hline 2 & 7 & 0.9848 & 5 & 8 & 0.9951 & 8 & 17 & 0.9638 & 14 & 15 & 0.9851 \\
\hline 2 & 8 & 0.9828 & 5 & 9 & 0.9748 & 8 & 18 & 0.9509 & 14 & 16 & 0.9808 \\
\hline 2 & 9 & 0.9628 & 5 & 10 & 0.9733 & 8 & 19 & 0.9504 & 14 & 17 & 0.9332 \\
\hline 2 & 10 & 0.9612 & 5 & 11 & 0.9855 & 8 & 20 & 0.9413 & 14 & 18 & 0.9324 \\
\hline 2 & 11 & 0.9727 & 5 & 12 & 0.9601 & 9 & 10 & 0.9793 & 14 & 19 & 0.9482 \\
\hline 2 & 12 & 0.9482 & 5 & 13 & 0.9555 & 9 & 11 & 0.9666 & 14 & 20 & 0.9441 \\
\hline 2 & 13 & 0.9436 & 5 & 14 & 0.9479 & 9 & 12 & 0.9474 & 15 & 16 & 0.9937 \\
\hline 2 & 14 & 0.9361 & 5 & 15 & 0.9545 & 9 & 13 & 0.9428 & 15 & 17 & 0.9407 \\
\hline 2 & 15 & 0.9426 & 5 & 16 & 0.9544 & 9 & 14 & 0.9354 & 15 & 18 & 0.9415 \\
\hline 2 & 16 & 0.9425 & 5 & 17 & 0.9601 & 9 & 15 & 0.9418 & 15 & 19 & 0.9591 \\
\hline 2 & 17 & 0.9482 & 5 & 18 & 0.9473 & 9 & 16 & 0.9418 & 15 & 20 & 0.9542 \\
\hline 2 & 18 & 0.9354 & 5 & 19 & 0.9468 & 9 & 17 & 0.9474 & 16 & 17 & 0.9417 \\
\hline 2 & 19 & 0.9350 & 5 & 20 & 0.9377 & 9 & 18 & 0.9347 & 16 & 18 & 0.9442 \\
\hline 2 & 20 & 0.9260 & 6 & 7 & 0.9941 & 9 & 19 & 0.9342 & 16 & 19 & 0.9636 \\
\hline 3 & 4 & 0.9888 & 6 & 8 & 0.9921 & 9 & 20 & 0.9253 & 16 & 20 & 0.9579 \\
\hline 3 & 5 & 0.9974 & 6 & 9 & 0.9719 & 10 & 11 & 0.9668 & 17 & 18 & 0.9636 \\
\hline 3 & 6 & 0.9974 & 6 & 10 & 0.9703 & 10 & 12 & 0.9492 & 17 & 19 & 0.9442 \\
\hline 3 & 7 & 0.9953 & 6 & 11 & 0.9817 & 10 & 13 & 0.9447 & 17 & 20 & 0.9579 \\
\hline 3 & 8 & 0.9932 & 6 & 12 & 0.9571 & 10 & 14 & 0.9372 & 18 & 19 & 0.9636 \\
\hline 3 & 9 & 0.9730 & 6 & 13 & 0.9525 & 10 & 15 & 0.9437 & 18 & 20 & 0.9867 \\
\hline 3 & 10 & 0.9715 & 6 & 14 & 0.9449 & 10 & 16 & 0.9436 & 19 & 20 & 0.9867 \\
\hline 3 & 11 & 0.9833 & 6 & 15 & 0.9515 & 10 & 17 & 0.9492 & & & \\
\hline 3 & 12 & 0.9583 & 6 & 16 & 0.9514 & 10 & 18 & 0.9365 & & & \\
\hline 3 & 13 & 0.9537 & 6 & 17 & 0.9571 & 10 & 19 & 0.9360 & & & \\
\hline 3 & 14 & 0.9461 & 6 & 18 & 0.9442 & 10 & 20 & 0.9270 & & & \\
\hline 3 & 15 & 0.9526 & & & & 11 & 12 & 0.9553 & & & \\
\hline \multicolumn{12}{|c|}{ Average reliability $=0.9620$} \\
\hline
\end{tabular}

$\mathrm{S}=$ Sender node, $\mathrm{D}=$ Destination Node, $\mathrm{R}=$ Reliability 